\title{
Desenvolvimento de um modelo de equações estruturais simultâneas para avaliação da qualidade de serviço em aeroportos
}

\author{
Development of simultaneous structural equations model to evaluate the \\ quality of service at airports
}

Eliézer Mello de Souza ${ }^{1}$, Anderson Ribeiro Correia $^{1}$

\section{RESUMO}

Este trabalho teve como objetivo desenvolver um modelo para avaliar a qualidade de serviço em aeroportos por meio de equações estruturais simultâneas. Para tanto, foram realizadas pesquisas na região de múltiplos aeroportos de São Paulo. Para a validação do modelo proposto neste trabalho, foram empregados dados obtidos em pesquisas no aeroporto de Congonhas. Foi adotada uma abordagem confirmatória ao problema de pesquisa, o que possibilitou a representação de modelo complexo das inter-relações entre os diversos fatores associados à percepção do passageiro sobre o nível de serviço e sua atitude perante o aeroporto. Os indicadores apresentados sobre a aderência do modelo demonstram que a metodologia proposta e as pesquisas de campo desenvolvidas foram adequadamente empregadas e modeladas, oferecendo um robusto modelo para que pesquisadores e profissionais da área o utilizem em análises específicas.

Palavras-chave: Transporte aéreo, Qualidade de serviço, Análise multivariada.

\begin{abstract}
This work aimed to develop a model to assess the quality of service at airports using simultaneous structural equations. To this end, surveys were conducted in the region of multiple airports in São Paulo. For validation of the proposed model in this work, data obtained at Congonhas Airport were used. A confirmatory approach was adopted to the research problem, which enabled the representation of a complex model of the interrelations between various factors associated with the passenger's perception of the level of service and their attitude towards the airport. The indicators presented about the adherence of the model demonstrate that the proposed methodology and the developed field researches were employed and modeled, offering a robust model for researchers and professionals in the field to use specific analyzes.
\end{abstract}

Keywords: Air transport, Quality of service, Multivariate analysis. 


\section{INTRODUÇÃO}

A análise de qualidade de serviço em aeroportos é um tema que tem envolvido pesquisadores, operadores aeroportuários e entidades governamentais relacionadas à administração e fiscalização do transporte aéreo. $\mathrm{O}$ ambiente aeroportuário no Brasil e no exterior tem sido marcado por crescente competição e privatização. Nesse sentido, oferecer um bom nível de serviço em todas as etapas de um voo comercial é uma preocupação constante, pois pode influenciar o volume de clientes e, portanto, as receitas aeroportuárias. Um potencial passageiro que convive na Grande São Paulo, por exemplo, desfruta várias opções para comprar uma passagem, podendo partir de Guarulhos, Congonhas e até mesmo de Viracopos. Enquanto no passado todos esses aeroportos eram administrados pela mesma empresa federal, a Infraero, hoje Guarulhos e Viracopos são administrados por empresas privadas e distintas. Logo, eles competem pelos clientes. Nessa mesma linha de raciocínio, boa parte dos aeroportos brasileiros é formada por empresas globais que possuem ações em bolsas de valores. A imagem que essas empresas possuem pode afetar seu desempenho no mercado financeiro. Com isso, é extremamente ruim que um aeroporto ofereça um serviço inadequado, pois sua imagem é também afetada.

Não é simples medir a qualidade de serviço, já que essa avaliação envolve fatores subjetivos e objetivos. Enquanto é relativamente simples avaliar o nível de serviço em um aeroporto com o uso de métricas espaciais ou temporais (m2/passageiro, tempo de espera em uma fila etc.), a avaliação de métricas ligadas à satisfação e como isso implica na atitude dos passageiros é um esforço complexo. Além disso, essa complexidade torna-se cada vez mais crescente quando se avaliam os múltiplos componentes de um aeroporto, lembrando que muitos deles são administrados por entidades diferentes, como empresas aéreas, operadores aeroportuários ou entidades governamentais como a Receita ou Polícia Federal.

$\mathrm{O}$ ambiente de globalização em que vivemos hoje é outro ponto que interfere bastante na medição da qualidade de serviço. Os passageiros que circulam nos aeroportos brasileiros são cada vez mais globalizados. A avaliação da qualidade de serviço de Congonhas e Guarulhos, por exemplo, é influenciada pela expectativa que os passageiros têm desse serviço, o que por sua vez é influenciada pelas comparações que fazem. Portanto, um passageiro que conhece bons aeroportos fora do Brasil, como de Cingapura, Dubai, Copenhagen ou Hong Kong, julgará o serviço prestado com muito mais rigor do que passageiros que raramente saem do país.

Nesse contexto de propor novos métodos para avaliação da qualidade de serviço, o objetivo deste trabalho foi analisar as relações entre expectativa, qualidade percebida do serviço, valor percebido, imagem do aeroporto, satisfação e lealdade e a atitude do passageiro em relação ao serviço aeroportuário. Para tanto, fez-se necessário explorar ferramentas multivariadas de análise de dados e aplicar pesquisas de campo para coleta de dados relevantes.

\section{REVISÃO DE LITERATURA}

Os primeiros trabalhos de avaliação de nível de serviço existentes na literatura datam de 1979, desenvolvidos pela Transport Canada ${ }^{1}$. De forma mais ampla, em 1987, um grupo de profissionais incentivados pela Administração Federal de Aviação dos Estados Unidos (Federal Aviation Administration - FAA) se reuniu para propor indicadores que pudessem ser amplamente empregados, buscando assim oferecer critérios minimamente aceitáveis em âmbito internacional ${ }^{2}$. Os critérios propostos foram importados das métricas empregadas para a avaliação do nível de serviço em rodovias, já que esta era bem mais avançada do que os trabalhos para a área aeroportuária. Foi essa a tônica da maior parte dos estudos não apenas nesse período, mas também nos anos subsequentes. Conforme relatado em Correia e Wirasinghe ${ }^{3}$, os trabalhos até então eram baseados em poucos indicadores, utilizavam pouca base de dados e focavam nos componentes individuais no aeroporto, deixando de oferecer métricas que avaliassem o aeroporto como uma organização coesa. Mesmo os trabalhos ligados às organizações aeroportuárias e de empresas aéreas tinham essas fragilidades.

Correia ${ }^{4}$ apresentou uma proposta inédita de avaliação da qualidade de serviço global de um aeroporto, trazendo algumas inovações, como a medição da satisfação e a avaliação completa do aeroporto. A ideia era avaliar o nível de serviço global em função do nível de serviço dos componentes individuais. Pesquisa de campo foi aplicada em aeroportos brasileiros, por meio de entrevistas com passageiros. Empregou-se a regressão linear para correlacionar os resultados pesquisados.

Dando um passo adiante, Bezerra e Gomes ${ }^{5}$ buscaram desenvolver a avaliação de qualidade de serviço multidimensional, em que não apenas a qualidade de serviço de um aeroporto era influenciada pela qualidade dos componentes individuais, mas estes também eram influenciados entre si. Para fazer frente a esse desafio, aplicaram ferramentas de análise de dados dinâmicos e multivariados, apresentando uma abordagem multidimensional para medição da qualidade de serviço em aeroportos baseada em práticas usuais do setor aeroportuário. Essa proposta ressaltou a importância da percepção da qualidade do serviço por parte do passageiro como fatorchave para orientar as decisões sobre o planejamento e a gestão do nível de serviço em aeroportos. O modelo de qualidade de serviço em aeroporto proposto por esses autores contemplou uma estrutura reflexiva para avaliação da qualidade de serviço e utilizou itens de medida usuais no setor.

Trabalhos semelhantes foram desenvolvidos mais recentemente, como o descrito em Allen et al. ${ }^{6}$, aplicando análise de componentes principais e equações estruturais simultâneas, com o objetivo de avaliar a qualidade de serviço em um aeroporto 
italiano, mesclando itens bastante diversos, como limpeza de banheiros, sinalização do terminal, assim como critérios objetivos ligados aos tempos de espera no check-in, controle de passaportes e segurança. A Fig. 1 apresenta a representação esquemática da influência da qualidade de serviços dos componentes específicos entre si e da qualidade de serviço geral, intitulada neste trabalho como OVSERVICE.

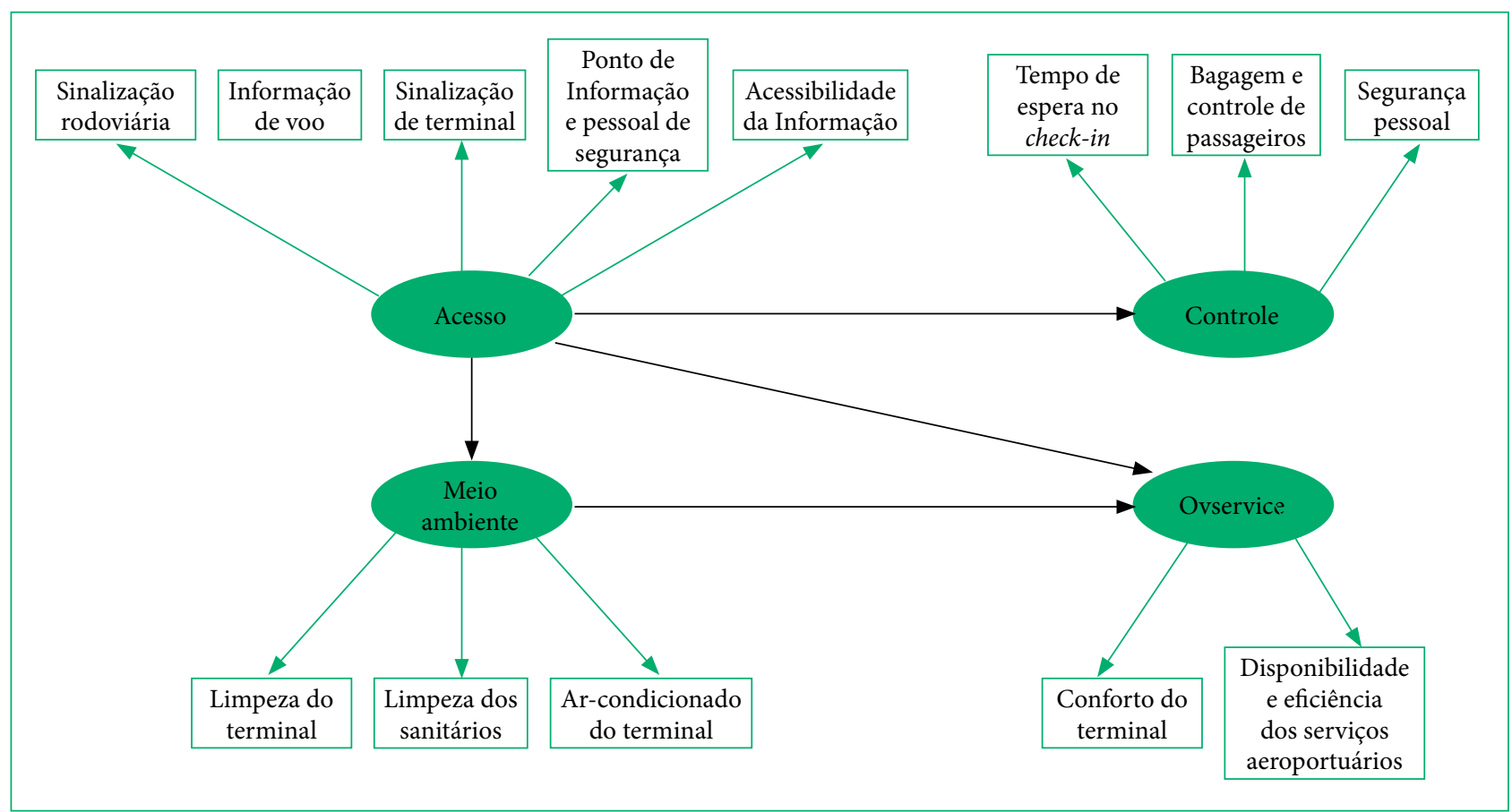

Figura 1: Modelo de avaliação de qualidade de serviço proposto por Allen et al.6.

Estudos similares foram possíveis mais recentemente com o desenvolvimento de ferramentas computacionais automatizadas e métodos de coleta de dados em campo com o apoio de ferramentas virtuais. Trata-se, portanto, de uma tendência recente. $\mathrm{Na}$ mesma linha de pensamento, Hong et al. ${ }^{7}$ exploram essas técnicas e buscam estratificar os resultados em função do tipo de cliente, seja ele o usuário do transporte aéreo, seja um prestador de serviço dentro do aeroporto. Nota-se que as avaliações foram pouco a pouco saindo do aspecto meramente espacial e temporal de avaliar um componente individual para a avaliação global de um aeroporto, envolvendo cada vez mais critérios e mesclando indicadores objetivos com subjetivos e técnicas de avaliação de satisfação de clientes.

Com o uso dessas técnicas, pretendemos avançar um pouco mais no conhecimento existente, buscando explorar como a qualidade de serviço é avaliada pela expectativa de passageiros e como pode gerar lealdade nos clientes, afetando na escolha por aeroportos, quando em uma região com múltiplos aeroportos.

\section{MODELO CONCEITUAL E HIPÓTESES}

A técnica empregada neste trabalho foi extraída do conjunto de ferramentas de análise multivariada. Particularmente, equações estruturais simultâneas foram utilizadas para a análise dos dados. Esse tipo de modelagem vem ganhando força na avaliação de qualidade de serviço em aeroportos, quando se consideram múltiplos indicadores e quando estes são influenciados entre si e também interferem nos indicadores globais, conforme demonstrado na revisão de literatura.

Hair et al. ${ }^{8}$ apresentam, de acordo com a Fig. 2, uma sequência para aplicação de um modelo de equações estruturais simultâneas que envolve:

- definição do modelo teórico;

- coleta de dados;

- expressão gráfica das relações de causa e efeito;

- ajuste dos modelos de mensuração e estrutural;

- identificação e avaliação do modelo;

- avaliação do modelo;

- modelo final. 


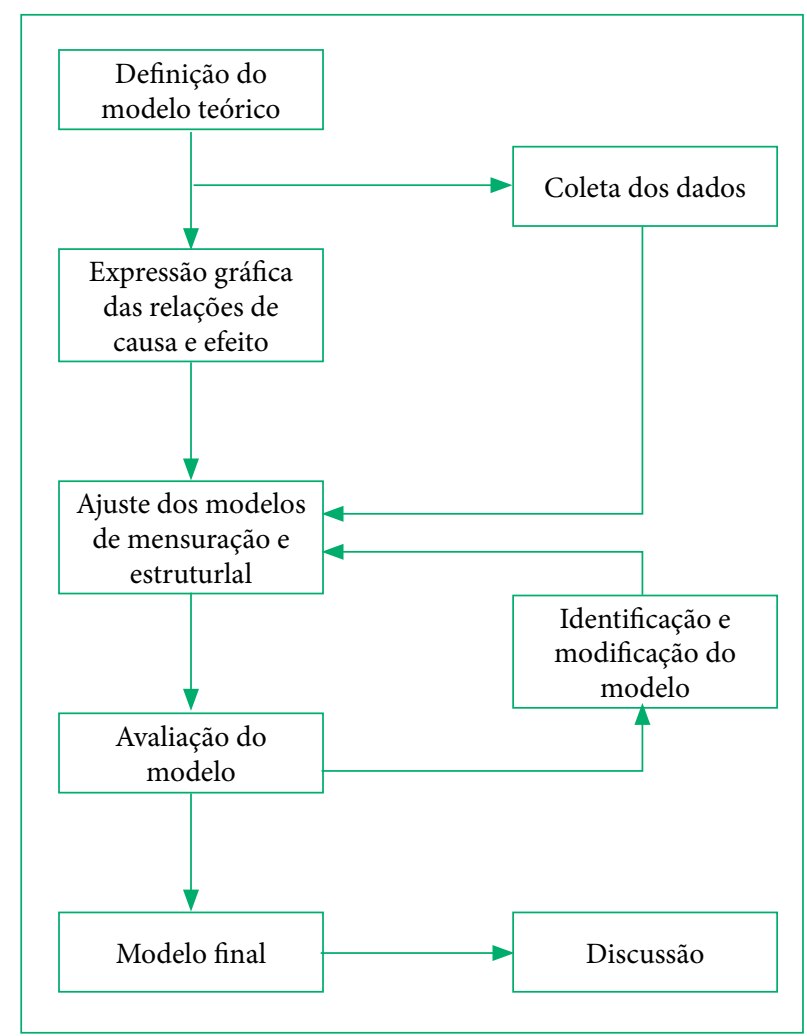

Figura 2: Etapas para a construção de um modelo de equações estruturais.

O modelo teórico proposto e os indicadores de qualidade foram extraídos da literatura, especificamente de Bezerra e Gomes ${ }^{5}$, que aplicaram um modelo europeu de avaliação de qualidade de serviço usado no mundo inteiro, já que aborda uma estrutura aeroportuária que é comum globalmente. A ideia de usar esse modelo e seus indicadores partiu de uma cooperação internacional entre Brasil e Portugal para a comparação da situação brasileira com a europeia. Portanto, seria importante utilizar as mesmas métricas para a comparação futura.

O presente modelo de satisfação é apresentado na Fig. 3. A satisfação do passageiro é o centro de uma cadeia entre as relações causais dos construtos antecedentes da satisfação do passageiro (expectativa, imagem de destino, qualidade percebida e valor percebido) e seu consequente (lealdade do passageiro). Foram utilizados indicadores reflexivos em que a variável latente é definida como uma variável que causa os indicadores observados e suas causadoras estão fora do modelo. A Tabela 1 traz um resumo desses indicadores, divididos em seis categorias principais.

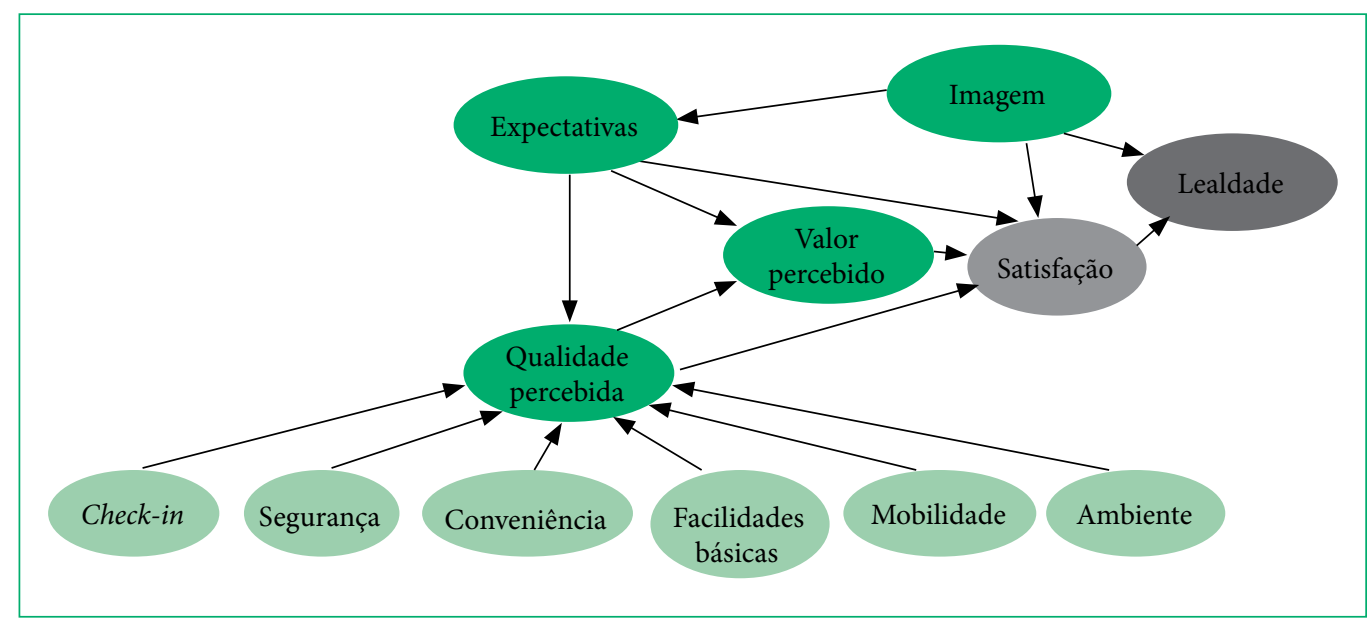

Figura 3: Modelo teórico simplificado. 
Tabela 1: Áreas/dimensões do questionário e seus componentes avaliados.

\begin{tabular}{|c|c|c|}
\hline Área & Descrição & Componentes \\
\hline A1 & Check-in & $\begin{array}{l}\text { Tempo de espera em fila; } \\
\text { Eficiência dos funcionários; } \\
\text { Atendimento e cortesia dos funcionários. }\end{array}$ \\
\hline A2 & Segurança & $\begin{array}{l}\text { Tempo de espera na fila da inspeção; } \\
\text { Rigor na inspeção; } \\
\text { Atendimento e cortesia dos funcionários da inspeção; } \\
\text { Sensação de estar protegido e seguro. }\end{array}$ \\
\hline A3 & Conveniência & $\begin{array}{c}\text { Restaurantes/instalações para alimentação; } \\
\text { Atendimento e cortesia dos funcionários dos restaurantes/instalações para alimentação; } \\
\text { Lojas e estabelecimentos comerciais; } \\
\text { Atendimento e cortesia dos funcionários das lojas/dos estabelecimentos comerciais; } \\
\text { Disponibilidade de bancos, caixas eletrônicos e casas de câmbio. }\end{array}$ \\
\hline A4 & Facilidades básicas & $\begin{array}{l}\text { Disponibilidade de banheiros; } \\
\text { Limpeza dos banheiros; } \\
\text { Conforto das áreas de espera/embarque. }\end{array}$ \\
\hline A5 & Mobilidade & $\begin{array}{l}\text { Facilidade de encontrar o seu caminho dentro do terminal; } \\
\text { Disponibilidade de painéis de informação de voos; } \\
\text { Distância percorrida a pé dentro do terminal; } \\
\text { Atendimento e cortesia dos funcionários do aeroporto (exceto check-in, segurança e área comercial). }\end{array}$ \\
\hline A6 & Ambiente & $\begin{array}{l}\text { Limpeza geral do aeroporto; } \\
\text { Conforto térmico no aeroporto; } \\
\text { Conforto acústico no aeroporto. }\end{array}$ \\
\hline
\end{tabular}

Dando sequência ao desenvolvimento da modelagem, a seção seguinte apresenta a coleta de dados especificamente para o aeroporto de Congonhas, em São Paulo.

\section{PESQUISA DE CAMPO}

Foi realizada uma primeira pesquisa no aeroporto de Congonhas, em dois dias do mês de outubro de 2015. Ao todo, foram entrevistados 482 passageiros, por uma equipe de cinco pesquisadores. Durante a pesquisa, tomou-se o cuidado de a abordagem ser feita de forma que a amostra fosse aleatória e evitasse o enviesamento. O pessoal que participou como pesquisador no aeroporto foi instruído sobre a abordagem. Ao passageiro abordado, primeiramente se perguntava se dispunha de tempo para colaborar com a pesquisa, explicava-se o questionário e, então, o passageiro recebia-o em uma prancheta para o preenchimento, estando o pesquisador à sua disposição para o caso de qualquer dúvida. De maneira geral, os passageiros queriam colaborar com a pesquisa, pois de algum modo achavam que poderiam colaborar para a melhoria dos serviços oferecidos.

O questionário aplicado envolvia a análise de dados socioeconômicos, experiência dos passageiros no aeroporto para a viagem atual e também viagens anteriores, local de residência e principalmente os dados de expectativa, que foram avaliados neste trabalho. Particularmente, para este último item, a Fig. 4 apresenta o resultado consolidado.

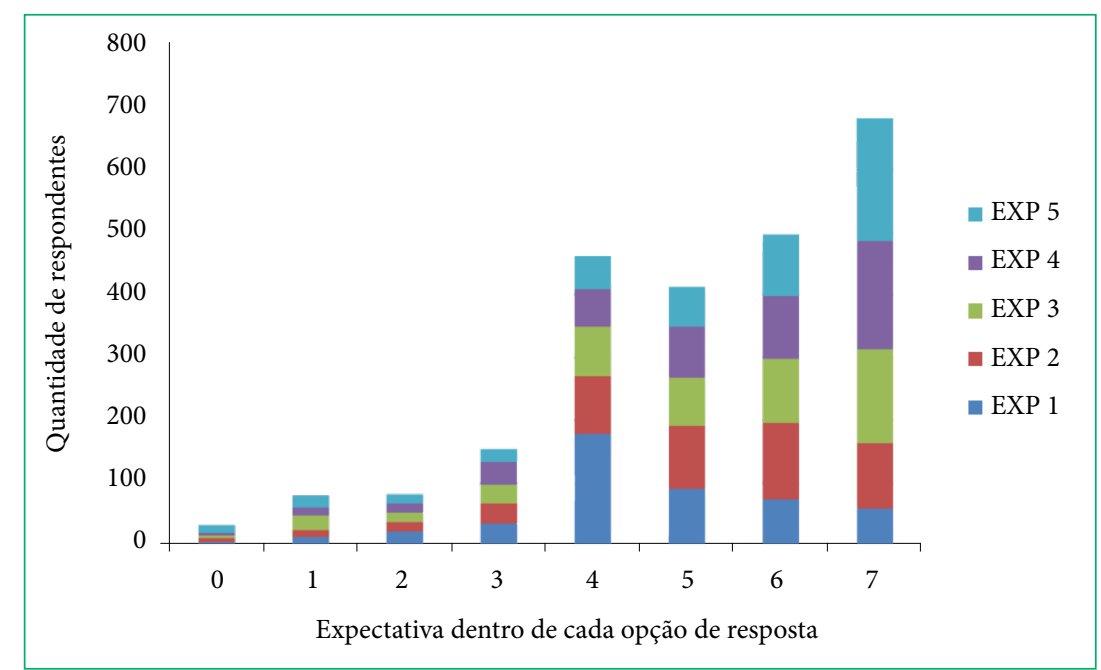

Figura 4: Expectativa do passageiro do aeroporto de Congonhas. EXP: expectativa. 
As respostas estão graduadas de 1 a 7, de forma que 1 significa discordo totalmente e 7 concordo totalmente. As expressões EXP1 a EXP5 significam o seguinte:

- EXP1: expectativa global sobre o aeroporto;

- EXP2: expectativa a respeito da capacidade de o aeroporto oferecer um serviço que atenda plenamente às necessidades;

- EXP3: expectativa acerca da possibilidade de falhas na prestação dos serviços;

- EXP4: expectativa quanto à rapidez e eficiência dos serviços;

- EXP5: expectativa em relação a conforto e segurança no aeroporto.

O número 0 identifica ausência de resposta por parte do pesquisado.

Esses dados de expectativa, assim como os outros indicadores socioeconômicos e da experiência do passageiro, foram empregados na modelagem, que será descrita nas próximas seções.

\section{CONSTRUÇÃO DO MODELO}

O modelo teórico foi comparado com o modelo criado com base nos dados levantados na pesquisa, para verificar se as relações descritas na teoria são de fato estabelecidas e se o modelo está devidamente ajustado.

Alterações nas relações do modelo foram feitas na medida do necessário, até que ele pudesse representar de maneira ajustada o modelo teórico proposto. Para verificar o ajustamento do modelo, utilizaram-se diversos índices, que serão apresentados oportunamente, os quais demonstram de maneiras diferentes o ajuste do modelo, sua excentricidade e a capacidade de representar o modelo teórico predefinido.

O modelo teórico tem como objetivo descrever atitudes do passageiro em relação ao aeroporto pesquisado. Dessa forma, utilizou-se o questionário já citado, composto de questões nas quais as respostas explicitam os seguintes fatores: expectativa (EXP), valor percebido (VAL), imagem (IMG), satisfação (SAT), lealdade (LOY) e qualidade percebida (QUA). Para melhor representação do modelo, o constructo QUA foi utilizado como constructo de segunda ordem, tendo sido representado no modelo pelos constructos check-in (CHK), segurança (SEC), mobilidade (MOB), conveniência (CON), facilidades básicas (BAS) e ambiente (AMB).

Partiu-se então do modelo apresentado, e foram feitos testes para encontrar o modelo mais ajustado possível. O modelo utiliza a configuração descrita a seguir, em que as variáveis foram extraídas da utilização de respostas fornecidas pela pesquisa de campo, em virtude do questionário aplicado:

- EXP: medido pelas questões EXP3, EXP4 e EXP5;

- VAL: medido pelas questões VAL1, VAL2 e VAL3;

- IMG: medido pelas questões IMG1, IMG2 e IMG3;

- SAT: medido pelas questões SAT1, SAT2, SAT3 e SAT5;

- LOY: medido pelas questões LOY1, LOY4 e LOY5;

- CHK: medido pelas questões CHK1, CHK2 e CHK3;

- SEC: medido pelas questões SEC1, SEC2, SEC3 e SEC4;

- MOB: medido pelas questões MOB1, MOB2 e MOB3;

- CON: medido pelas questões CON1, CON2, CON3, CON4 e CON5;

- BAS: medido pelas questões BAS1, BAS2 e BAS3;

- AMB: medido pelas questões AMB1, AMB2 e AMB3;

Para as análises e a construção dos gráficos, o software Amos foi empregado, o qual é amplamente utilizado no mundo todo para o desenvolvimento de análises semelhantes. O diagrama de caminhos para o modelo teórico definido está representado na Fig. 5. Esse diagrama é derivado do questionário que apresenta 11 constructos ou variáveis latentes - EXP, VAL, IMG, SAT, LOY, CHK, SEC, MOB, CON, BAS e AMB -, medidos pelas suas respectivas perguntas. Todos os itens que são variáveis observadas (medidas) possuem associados a si um erro, que representa o erro de medida. Os 11 fatores são correlacionados, como pode ser visto por meio das setas bidirecionais entre todos os fatores. Há setas unidirecionais apontando os fatores em direção às suas respectivas variáveis observadas, indicando que os valores dessas variáveis são influenciados pelos seus respectivos fatores. Já as setas unidirecionais, oriundas dos erros, representam o impacto do erro de medida nas variáveis observadas. As setas bidirecionais são representativas das correlações (ou covariâncias) entre os fatores (variáveis latentes). 


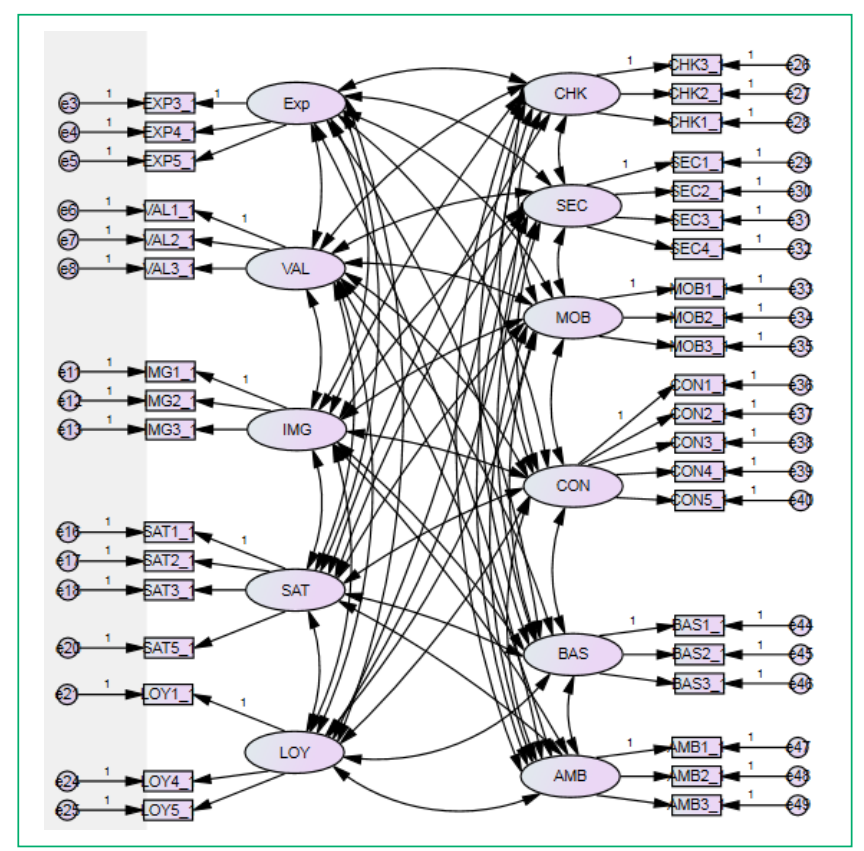

Figura 5: Diagrama de caminhos para o modelo proposto.

Os componentes do modelo são descritos como segue:

- Variáveis latentes: EXP, VAL, IMG, SAT, LOY, CHK, SEC, MOB, COM, BAS e AMB;

- Variáveis observadas: EXP3..., EXP5, VAL1..., VAL3, IMG1.., IMG3, SAT1..., SAT3, SAT5, LOY1, LOY4, LOY5, CHK1..., CHK3, SEC1.., SEC4, MOB1.., MOB3, CON1.., CON5, BAS1 .., BAS3, AMB1..., AMB3;

- Erros de medida: e3..., e5, e6..., e8, e11.., e13, e16.., e18, e20, e21, e24, e25, e26.., e28, e29..., e32, e33..., e35, e36..., e40, e44..., e46, e47..., e49.

De forma geral, as equações podem ser representadas matricialmente pela Eq. 1:

$$
\mathrm{Y}=\Lambda \mathrm{y} \eta+\varepsilon
$$

em que: $\mathrm{Y}=$ a matriz dos indicadores (variável observada); $\Lambda \mathrm{y}=$ a matriz $\mathrm{p} \times \mathrm{m}$ dos coeficientes da variável latente; $\eta=\mathrm{o}$ vetor $\mathrm{m} \times 1 \mathrm{da}$ variável latente; $\varepsilon=0$ vetor $\mathrm{p} \times 1$ de erro de medição dos indicadores.

No caso estudado em questão, as equações do modelo de medida podem ser representadas pelas Eqs. de 2 a 12:

- Para o constructo EXP:

em que: $\mathrm{i}=[3,4,5] ; \mathrm{k}=[3,4,5]$.

$$
\mathrm{EXPi}=\lambda \mathrm{i} \mathrm{EXP}+\mathrm{ek}
$$

- Para o constructo VAL:

em que: $i=[3,4,5] ; k=[6,7,8]$.

$$
\mathrm{VALi}=\lambda \mathrm{i} \mathrm{VAL}+\mathrm{ek}
$$

- Para o constructo IMG:

$$
\mathrm{IMGi}=\lambda \mathrm{i} \mathrm{IMG}+\mathrm{ek}
$$

em que: $\mathrm{i}=[1,2,3] ; \mathrm{k}=[11,12,13]$.

- Para o constructo SAT:

em que: $\mathrm{i}=[1,2,3] ; \mathrm{k}=[16,17,18]$.

$$
\mathrm{SATi}=\lambda \mathrm{i} \mathrm{SAT}+\mathrm{ek}
$$

- Para o constructo LOY:

$$
\mathrm{LOY} \mathrm{i}=\lambda \mathrm{i} \mathrm{LOY}+\mathrm{ek}
$$

em que: $\mathrm{i}=[4,5] ; \mathrm{k}=[24,25]$. 
- Para o constructo CHK:

$$
\mathrm{CHK} \mathrm{i}=\lambda \mathrm{i} \mathrm{CHK}+\mathrm{ek}
$$

em que: $\mathrm{i}=[1,2,3] ; \mathrm{k}=[26,27,28]$.

- Para o constructo SEC:

em que: $\mathrm{i}=[1,2,3,4] ; \mathrm{k}=[29,30,31,32]$.

$$
\text { SEC } \mathrm{i}=\lambda \mathrm{i} \text { SEC }+\mathrm{ek}
$$

- Para o constructo MOB:

em que: $\mathrm{i}=[1,2,3] ; \mathrm{k}=[33,34,35]$.

$$
\mathrm{MOB} i=\lambda \mathrm{i} \mathrm{MOB}+\mathrm{ek}
$$

- Para o constructo CON:

em que: $\mathrm{i}=[1,2,3,4,5] ; \mathrm{k}=[36,37,38,39,40]$.

$$
\mathrm{CON} \mathrm{i}=\lambda \mathrm{i} \mathrm{CON}+\mathrm{ek}
$$

- Para o constructo BAS:

em que: $\mathrm{i}=[1,2,3] ; \mathrm{k}=[44,45,46]$.

$$
\mathrm{BAS} \mathrm{i}=\lambda \mathrm{i} \mathrm{BAS}+\mathrm{ek}
$$

- Para o constructo AMB:

em que: $\mathrm{i}=[1,2,3] ; \mathrm{k}=[47,48,49]$.

$$
\mathrm{AMB} i=\lambda \mathrm{i} A M B+\mathrm{ek}
$$

A identificabilidade consiste em verificar a existência de limitações explanatórias do modelo para gerar soluções únicas. O problema está centrado na transposição da matriz das variáveis observadas em parâmetros estruturais do modelo em estudo. Se há uma única solução encontrada para os valores dos parâmetros estruturais, o modelo é considerado identificado. Busca-se que o modelo seja superidentificado, isto é, que o número de parâmetros a serem estimados seja menor que o de variâncias e covariâncias das variáveis observadas. Isso resulta em graus de liberdade positivos, o que permite o teste do modelo.

Ainda com relação à identificação, existe também a necessidade de se definir uma escala para cada variável latente, pois são variáveis não observadas, não possuindo escala métrica definida. Esse requisito pode ser satisfeito utilizando um contraste, ou seja, um valor diferente de $0^{9}$ em cada uma das cargas fatoriais usadas para medir um mesmo fator; normalmente o valor empregado é 1 .

Diversos testes foram aplicados para avaliação da aderência do modelo como verificação da identificabilidade do modelo estrutural e avaliação dos critérios de ajuste do modelo, e encontraram-se bons resultados, com base em critérios apresentados pela literatura.

Como forma final de avaliação de ajuste do modelo, foi realizado o cálculo dos índices de modificação (modification índices - MI), que indica a queda mínima do valor de $\chi^{2}$ em duas situações: uma considerando que a variável seja retirada do modelo, outra caso o parâmetro listado fosse incluído no modelo, ou seja, fosse livremente estimado, fazendo-se a correlação indicada. Os resultados são apresentados nas Figs. 6 e 7. Os valores a serem considerados são sempre os maiores valores de MI, pois são os que mais diminuem o valor de $\chi^{2}$. A coluna par change representa o valor da estimativa desse novo parâmetro no modelo. Nas estimativas de modelos iniciais o valor de MI era de 149,635. O valor obtido a seguir representa a melhoria dos resultados do modelo, com seu ajuste consolidado. O maior valor encontrado de MI no modelo atual está em 32,428. Ainda é possível fazer alguma melhoria nos índices, mas da forma como o modelo se encontra já se pode passar à fase de modelagem estrutural e análise.

Na evolução dos modelos, optou-se por uma abordagem mais conservativa, não realizando a correlação dos erros, mas sim retirando-se as variáveis observadas com índices não aceitáveis, preservando-se no mínimo a quantidade de três variáveis observadas pelas variáveis latentes relacionadas, para não perder a qualidade da análise em cada variável latente.

A retirada ou não de determinada variável foi avaliada individualmente. No atual estágio do modelo há ainda a possibilidade de melhoria nos indicadores, contudo a retirada de variáveis é agora analisada sob a relação custo $\times$ benefício entre aperfeiçoamento dos indicadores e menor quantidade de variáveis observadas para análise. Chegou-se a um ponto que foi notado que a melhoria dos indicadores se apresenta numa ordem incremental muito pequena, tal que justifique a perda da variável observada para utilização no modelo estrutural e análise. 


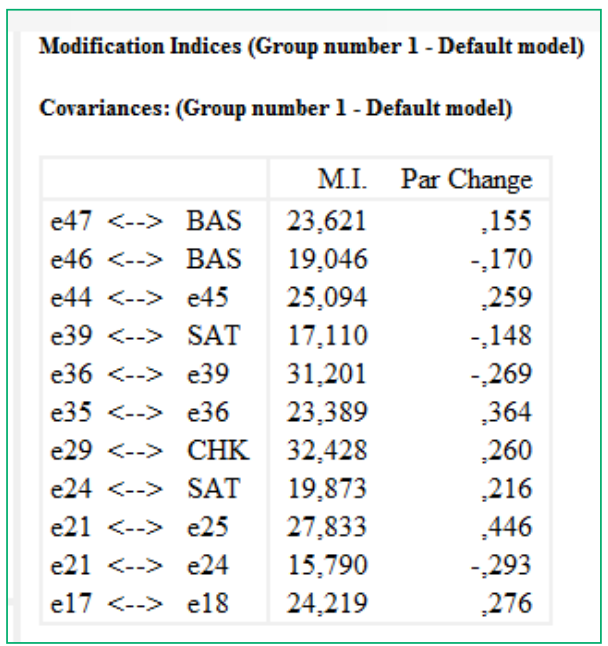

Figura 6: Índices de modificação (erros de medida).

\begin{tabular}{|c|c|c|c|c|}
\hline \multicolumn{5}{|c|}{$\begin{array}{l}\text { Modification Indices (Group number } 1 \text { - Default n } \\
\text { Covariances: (Group number } 1 \text { - Default model) }\end{array}$} \\
\hline & & & M.I. & Par Change \\
\hline e_amb2 & $<-->$ & e_amb3 & 88,295 &, 761 \\
\hline e_amb1 & $<-->$ & e_amb2 & 84,138 &, 620 \\
\hline e_bas 2 & $<-->$ & e_amb1 & 76,281 &, 601 \\
\hline e_bas1 & $<-->$ & e_bas 2 & 130,313 & 859 \\
\hline e_con6 & $<-->$ & e_con 7 & 107,888 & 1,160 \\
\hline e_mob1 & $<-->$ & e_mob2 & 100,455 &, 926 \\
\hline e_sec 2 & $<-->$ & e_sec 3 & 70,376 &, 613 \\
\hline e_sec1 & $<-->$ & e_sec 2 & 83,909 &, 742 \\
\hline e_chk2 & $<-->$ & e_sec1 & 75,776 &, 657 \\
\hline e_chk2 & $<-->$ & e_chk3 & 149,635 & 805 \\
\hline e_chk1 & $<-->$ & e_chk2 & 137,528 & 918 \\
\hline e_con3 & $<-->$ & e_con 4 & 121,589 & 643 \\
\hline e_con 3 & $<-->$ & e_con 1 & 79,837 & .637 \\
\hline
\end{tabular}

Figura 7: Índices de modificação (variáveis observadas).

\section{CONCLUSÕES}

Esta pesquisa apresentou um modelo inédito em desenvolvimento no Brasil, em que a avaliação da qualidade de serviço em aeroportos é proposta considerando a satisfação dos passageiros em função da expectativa. A lealdade dos passageiros é uma variável sugerida, demonstrando se a qualidade de serviço impacta na escolha e na quantidade de viagens que o passageiro efetua por meio de um aeroporto específico. Como bons resultados de aderência do modelo foram apresentados, os próximos estudos serão feitos com maior segurança. Pesquisas adicionais estão sendo desenvolvidas, no intuito de verificar se existe alguma variação nos resultados quando contrastados com diferentes segmentos do universo pesquisado, como, por exemplo, passageiros frequentes e não frequentes e viagens feitas a negócio ou por outro motivo. Esses resultados devem ser consolidados em próximos artigos.

\section{AGRADECIMENTOS}

Os autores deste trabalho gostariam de agradecer à Coordenação de Aperfeiçoamento de Pessoal de Nível Superior (CAPES), ao Conselho Nacional de Desenvolvimento Científico e Tecnológico (CNPq) e à Secretaria de Aviação Civil do Ministério dos Transportes o apoio providenciado no desenvolvimento das pesquisas.

\section{REFERÊNCIAS}

1. Transport Canada. A discussion paper on level of service definition and methodology for calculating airport capacity. Report TP 2027. Canadá: Transport Canada; 1979. 
2. Transportation Research Board. Measuring airport landside capacity. Special Report 215. Washington, D.C.: Transportation Research Board, National Research Council; 1987.

3. Correia AR, Wirasinghe SC. Evaluation of level of service at airport passenger terminals: a review of research approaches. Transportation Research Record 1888. Washington, D.C.: National Research Council; 2004.

4. Correia AR, Wirasinghe SC, Barros AG. A Global index for level of service evaluation at airport passenger terminals. Transp Res Part E. 2008;44(4):607-20. https://doi.org/10.1016/j.tre.2007.05.009

5. Bezerra GL, Gomes CF. Measuring airport service quality: a multidimensional approach. J Air Transp Manag. 2016;53:85-93. https://doi. org/10.1016/j.jairtraman.2016.02.001

6. Allen J, Bellizzi MG, Eboli L. Latent Factors on the assessment of Service Quality in an Italian Pefipheral Airport. 22nd Euro Working Group on Transportation Meeting; 2019; Barcelona. 2020.

7. Hong S, Choi D, Chae J. Exploring different airport users' service quality satisfaction between service providers and air travelers. J Retail Consum Serv. 2020;52:101917. https://doi.org/10.1016/j.jretconser.2019.101917

8. Hair JF, Black WC, Babin B, Anderson RE, Tatham RL. Multivariate Data Analysis. 6ª ed. Upper Saddle River: Prentice-Hall; 2005

9. Byrne BM. Structural equation modeling: perspectives on the present and the future. Int J Testing. 2001;1(3-4):327-34. https://doi.org/10.108 0/15305058.2001.9669479 